\title{
Effects of Curing Temperature on the Optical and Charge Trap Properties of InP Quantum Dot Thin Films
}

\author{
Priyaranjan Mohapatra, Mai Xuan Dung, Jin-Kyu Choi, Sohee Jeong, ${ }^{\dagger}$ and Hyun-Dam Jeong* \\ Department of Chemistry and Institute of Basic Science, Chonnam National University, Gwangju 500-757, Korea \\ *E-mail: hdjeong@chonnam.ac.kr \\ ${ }^{\dagger}$ Nanomechanical Systems Research Division, Korea Institute of Machinery and Materials, Daejeon 305-343, Korea \\ Received September 29, 2010, Accepted November 16, 2010
}

\begin{abstract}
Highly luminescent and monodisperse InP quantum dots (QDs) were prepared by a non-organometallic approach in a non-coordinating solvent. Fatty acids with well-defined chain lengths as the ligand, a non coordinating solvent, and a thorough degassing process are all important factors for the formation of high quality InP QDs. By varying the molar concentration of indium to ligand, QDs of different size were prepared and their absorption and emission behaviors studied. By spin-coating a colloidal solution of InP QD onto a silicon wafer, InP QD thin films were obtained. The thickness of the thin films cured at 60 and $200{ }^{\circ} \mathrm{C}$ were nearly identical (approximately $860 \mathrm{~nm}$ ), whereas at $300{ }^{\circ} \mathrm{C}$, the thickness of the thin film was found to be $760 \mathrm{~nm}$. Different contrast regions (A, B, C) were observed in the TEM images, which were found to be unreacted precursors, InP QDs, and indium-rich phases, respectively, through EDX analysis. The optical properties of the thin films were measured at three different curing temperatures $\left(60,200,300{ }^{\circ} \mathrm{C}\right)$, which showed a blue shift with an increase in temperature. It was proposed that this blue shift may be due to a decrease in the core diameter of the InP QD by oxidation, as confirmed by the XPS studies. Oxidation also passivates the QD surface by reducing the amount of $\mathrm{P}$ dangling bonds, thereby increasing luminescence intensity. The dielectric properties of the thin films were also investigated by capacitance-voltage $(\mathrm{C}-\mathrm{V})$ measurements in a metal-insulator-semiconductor (MIS) device. At 60 and $300^{\circ} \mathrm{C}$, negative flat band shifts $\left(\Delta \mathrm{V}_{\mathrm{fb}}\right)$ were observed, which were explained by the presence of $\mathrm{P}$ dangling bonds on the InP QD surface. At $300^{\circ} \mathrm{C}$, clockwise hysteresis was observed due to trapping and detrapping of positive charges on the thin film, which was explained by proposing the existence of deep energy levels due to the indium-rich phases.
\end{abstract}

Key Words: InP, Quantum dot, Thin films, Charge trap

\section{Introduction}

Quantum dots (QDs) are nanometer scale particles that are neither small molecules nor bulk solids. Their composition and small size (a few hundreds to a few thousand atoms) give these dots extraordinary optical properties that can be readily customized by changing their size or composition. The quantum size effect occurs when the de Broglie wavelength of the valence electrons is of the same order as the size of the particle itself. Then, the particles behave electronically as zero-dimensional quantum dots (or quantum boxes). Since the key feature in QDs lies in their optical properties, governed by quantum confinement effects, it is therefore important to control nanocrystal size, distribution, crystallinity, and stoichiometry of the doped nanocrystals. In recent years, semiconductor nanoparticles (QDs) have been of great interest due to their optical properties, which are governed by the quantum confinement effect. These semiconductor nanoparticles (QDs) have versatile applications, such as single electron transistors, lasers, LEDs, ${ }^{1}$ biolabeling, bioimaging, ${ }^{2}$ and photovoltaic devices. ${ }^{3}$

Among the semiconductor nanocrystals, the commercially available group II-VI QDs like CdSe exhibit good photo stability, quantum yield, and more important, a tunable emission wavelength. ${ }^{4}$ Nevertheless, due to their intrinsic toxicity, research on II-VI semiconductor QDs remains limited. Therefore III-V QDs, particularly nitrides and phosphides, have been found to be the most desirable alternative due to their reduced toxi- city, large excitation diameter, and covalent character. Among the III-V QDs, InP QDs were more studied in terms of their synthesis. ${ }^{5}$ However, nucleation and crystal growth is time-consuming, generally requiring three to seven days, and the quantum yield are typically very low. For the first time, Peng et al. reported an excellent method to synthesize InP QDs in a noncoordinating solvent, which was very fast and controllable. ${ }^{6}$ In these methods the indium precursor was heated with fatty acids of different chain lengths. By varying the indium to fatty acid molar ratio, the size of the quantum dots could be varied, indicating that the carboxylate groups act as an in situ selective coordination ligand.

Like the semiconductor, QDs can be viewed as "artificial atoms"; similarly, a superlattice of nanocrystals by analogy can be referred to as artificial solids. This artificial solid can be given different terminologies, such as QD arrays, QD superlattice, QD solids, or QD thin films. In this article, the authors will employ QD solids. Typically, solids can be classified into metals, insulators, and semiconductors, depending upon their electronic properties, but there is a very limited degree of control as the fundamental unit of these solids, the atom, transcends modification. Whereas in the case of nanocrystal solids, the fundamental unit is a quantum dot, which has can be tuned in a number of configurations because of associated degrees of freedom. Furthermore, it is possible to tune the interparticle coupling of quantum dots by modifying size, as well as length and electronic structure of the surface ligands. An in-depth study of 
the formation of quantum dot solids or thin films is provided by Talapin et al.. ${ }^{7}$ QD solids with controlled and variable electron densities can be used in optoelectronic switches, LEDs, lasers, and solar cells. The major challenge to use semiconductor quantum dots in optoelectronic device is the poor charge transport properties of QD films, as the hopping mechanism ${ }^{8}$ is responsible for transport of charge between the QDs. Accordingly, the electronic nature of the capping ligand is very important to the transport properties of the QDs. Crystallization of colloidal QD solution samples into long-range ordered solids by spincoating requires precise control of particle size, monodispersity, and capping ligand, in addition to careful selection of the dispersing solvent and adjustment of the temperature affecting the rate of solvent evaporation. In this lab, the electronic interactions within the QD solids from the spin-coated CdS thin film transistors has already been investigated, along with study of tunable refractive indices and optical band gaps in oxidized Si QD solids. ${ }^{9}$

The possibilities of controlled assembly of crystalline solids from QDs open a new era for fundamental research, engineering of advanced materials, and the structural and physical characterization of QD solids. Among the III-V QDs, InP QD poses as a promising candidate for electronic devices such as hetero junction bipolar transistors (HBT), metal-insulator-semiconductor field effect transistors (MISFETs), microwave diodes, optoelectronic device, sensors, and data storage devices. ${ }^{10} \mathrm{Al}$ though recent advances have been made in the synthesis of crystalline InP QDs in colloidal solution that exhibit high quantum efficiency, the major challenge to use the QDs in an optoelectronic device is the availability of QD thin films on a substrate in large quantities and at a low cost.

There are very few papers available regarding the formation of InP quantum dot solid and their optical and electrical properties. Arthur Nozik and his group studied the optical and structural properties of uncoupled and close-packed arrays of InP quantum dots by solvent evaporation of a colloidal solution of InP QDs onto a sapphire substrate. ${ }^{11}$ However, the effects of curing temperature on the optical properties of the InP QD solid, as well as on electrical properties, has not yet been reported. In this article, the authors first discuss size control synthesis of InP QD colloids. This is followed by demonstration of a simple method for the preparation of InP QD thin films on a silicon substrate. The investigation focused primarily upon the effects of curing temperature on film properties such as optical absorption, photoluminescence, thickness, and charge trap properties.

\section{Experimental Section}

Materials. Indium (III) acetate $(99.99 \%$, Sigma, St Louis, MO, U.S.A.), myristic acid (99 - 100\%, Sigma), tris(trimethylsilyl) phosphine, (98\%, JSI silicone, Seongnam-si, Gyeonggi-do, South Korea), and 1-octadecene (90\%, Aldrich, St Louis, MO, U.S.A.) were used without further purification. Hexane and acetone were purchased from Dae Jung Chemicals \& Metals Co. Ltd. (Shiheung city, Gyeonggi-do, South Korea).

Synthesis Procedures. Synthesis of InP Quantum Dots. InP was synthesized with indium acetate, tris(trimethylsilyl)phos- phine $\left(\mathrm{P}(\mathrm{TMS})_{3}\right)$, and myristic acid (MA) as the indium and phosphorous precursor, and stabilizers, respectively, using the procedure proposed by Peng et al.. ${ }^{6}$ For a typical synthesis, 0.2 $\mathrm{mM}$ of indium acetate was mixed with $0.7 \mathrm{mM}$ myristic acid and $5.0 \mathrm{~mL}$ of octadecene in a three-neck flask fitted with a condenser under argon atmosphere. The flask was heated to $120^{\circ} \mathrm{C}$ over a round bottom heating mantle filled with sand to obtain an optically clear solution. Then, the flask was repeatedly evacuated and refilled with argon for $2 \mathrm{~h}$ to provide a water and oxygen free atmosphere. The solution was quickly heated to $280^{\circ} \mathrm{C}$ under agitation. When the temperature of the solution became stable, $0.1 \mathrm{mM}$ solution of $\mathrm{P}(\mathrm{TMS})_{3}$ in $1.5 \mathrm{~mL}$ of octadecene was then rapidly injected. The solution was kept at $260{ }^{\circ} \mathrm{C}$ for $20 \mathrm{~min}$, and then quickly cooled to room temperature; an equivalent amount of hexane was then added. The precipitate was centrifuged at $5000 \mathrm{rpm}$ for $5 \mathrm{~min}$ and discarded. The clear quantum dots solution was collected. Acetone was added until the solution became turbid. The precipitate quantum dots were separated by centrifugation at $10,000 \mathrm{rpm}$ for $10 \mathrm{~min}$. The precipitates were dispersed in hexane for further characterization. This was repeated several times to separate the myristic acidcaped InP QDs from the solvent and byproducts.

\section{Analysis of InP Quantum Dots.}

UV-vis Absorbance Spectroscopy: The absorbance spectra of the InP QD colloidal solution dispersed in hexane were measured using a SCINCO- S-3150 spectrophotometer.

Photoluminescence Spectroscopy: Photoluminescence spectroscopy was measured using a He-Cd (Kimmon Electric Co., IK3501R-G, Itabashi-Ku, Tokyo, Japan) light source at $325 \mathrm{~nm}$ with $55 \mathrm{~mW}$ power and an intensified photodiode array detector (IRY1024, Princeton Instrument Co., Trenton, NJ, U.S.A.) to investigate the fluorescence properties of the InP QD solution.

X-Ray Diffraction (XRD): X-ray diffraction was studied on a RIGAKU D/Max-2500 X-ray diffractometer equipped with a $\mathrm{Cu} \mathrm{K \alpha}$ source at $40 \mathrm{kV}$ and $300 \mathrm{~mA}$. Samples for analysis were prepared on a silicon substrate by spin-coating. The $2 \theta$ angle was varied from $3^{\circ}$ to $60^{\circ}$ at a rate of $2^{\circ} / \mathrm{min}$.

Transmission Electron Microscopy: Transmission electron microscopy (TEM) was measured with a model 200 JEOL microscope at an acceleration voltage of $200 \mathrm{kV}$. The specimens were prepared by drop-casting the hexane dispersion onto a carbon-coated 300 mesh copper grid.

Preparation of InP Quantum Dot Thin Films. The 8 wt \% InP QD solution in hexane was filtrated (PTFE, $0.25 \mu \mathrm{m}$ ) and spin-coated on p-type Si (100) wafers (resistivity: $1-30 \Omega \cdot \mathrm{cm}$, thickness: $525 \mathrm{~nm}$ ) at $2000 \mathrm{rpm}$. The Si wafers were purchased from QL Electronics Co. Ltd, Ningbo, China and the resistivity values obtained from the same company. The thin films on the $\mathrm{Si}$ wafers were heated at $60{ }^{\circ} \mathrm{C}$ on a hot plate for $20 \mathrm{~min}$ in an open atmosphere to remove all remaining solvent. Finally, the films were cured for $30 \mathrm{~min}$ at 200 and $300{ }^{\circ} \mathrm{C}$ under a vacuum of approximately $1.0 \times 10^{-2}$ torr.

\section{Characterization of Quantum Dot Thin Films.}

X-Ray Photoelectron Spectroscopy: To study the change in composition of the thin film by changing the curing temperature from 60 to $300{ }^{\circ} \mathrm{C}, \mathrm{X}$-ray photoelectron spectroscopy (XPS) measurements were conducted on a multi lab 2000 using a $\mathrm{Mg}$ $\mathrm{K} \alpha(1253.6 \mathrm{eV})$ source at a pass energy of $20 \mathrm{eV}$ under a pre- 
ssure of $1.0 \times 10^{-9}$ Torr. An $\mathrm{Ar}^{+}$ion gun sputtering of 2 min at a power of $2 \mathrm{kV}$ and $1.3 \mu \mathrm{A}$ was used for a brief cleaning of the surface of the sample prior to XPS measurements.

UV-vis Absorbance Spectroscopy: The 8 wt \% InP QD solution in hexane was filtrated (PTFE, $0.25 \mu \mathrm{m}$ ) and spin-coated onto a quartz plate at $2000 \mathrm{rpm}$. The thin films on quartz plate were heated at $60^{\circ} \mathrm{C}$ on a hot plate for $20 \mathrm{~min}$ in an open atmosphere to remove the remaining solvents. Finally, the films were cured for $30 \mathrm{~min}$ at 200 and $300{ }^{\circ} \mathrm{C}$ under a vacuum of approximately $1.0 \times 10^{-2}$ torr. Then UV-visible spectra of these thin films were performed in a range of $400-8000 \mathrm{~nm}$ using a SCINCO-S-3150 spectrophotometer.

Photoluminescence Spectroscopy: The photoluminescence spectra of the thin films cured at 60,200 , and $300{ }^{\circ} \mathrm{C}$ were performed using a He-Cd (Kimmon Electric Co., IK3501R-G, City, Japan) light source at $325 \mathrm{~nm}$ with $55 \mathrm{~mW}$ power and an intensified photodiode array detector (IRY1024, Princeton Instrument Co.).

Transmission Electron Microscopy and Energy-dispersive X-ray Analyses. Transmission electron microscopy (FRTEM, FEI, Tecnai F30 Super-Twin, Eindhoven, Netherlands) was performed to investigate the microstructure of the thin films. Cross-sectional TEM specimens were prepared through mechanical polishing, followed by Ar ion-milling (GATAN, PIPS 691) for electron transparency. Energy-dispersive X-ray (EDX) analyses were made using a Tecnai 20 (FEI Company, Eindhoven, Netherlands) transmission electron microscope operated at an acceleration voltage of $300 \mathrm{kV}$ with an EDX/TEM HAADF (transmission electron microscope with a high-angle annular dark field) detector.

\section{Characterization of Electrical Properties.}

MIS Structures: Metal-insulator-semiconductor (MIS) device structures were fabricated to investigate the capacitance-voltage (C-V) characteristics for films cured at 60,200 , and $300{ }^{\circ} \mathrm{C}$. Aluminum metal was deposited onto the InP QD thin films by a thermal evaporation technique and served as a top electrode. The backside of the silicon wafer in the MIS devices was also coated with aluminum metal to diminish contact resistance. Using an HP4284 LCR meter, the C-V measurements were obtained by applying an ac voltage with a frequency of $1 \mathrm{MHz}$ and an amplitude of $100 \mathrm{mV}$ to the top aluminum electrode, and a DC bias voltage was swept over the range of -5 to $5 \mathrm{~V}$. C-V curves were acquired in both forward and reverse directions; afterward, these were compared with each other to study the charge trap mechanisms.

\section{Results and Discussion}

Synthesis and Characterization of InP Quantum Dots. Highly luminescent monodispersed InP QDs were prepared by the reaction of indium acetate with tris(trimethylsilyl phosphine) $\left(\mathrm{P}(\mathrm{TMS})_{3}\right)$ using myristic acid (MA) as the ligand and octadecene as the solvent as depicted in Fig. 1. Use of a strong coordinating ligand, such as trioctylphosphineoxide or trioctyl phosphine, caused unstable initial nuclei and more intrinsic defects. ${ }^{12}$ Therefore, use of indium carboxylate in a noncoordinating solvent could minimize the disadvantages of a strong coordinating ligand.

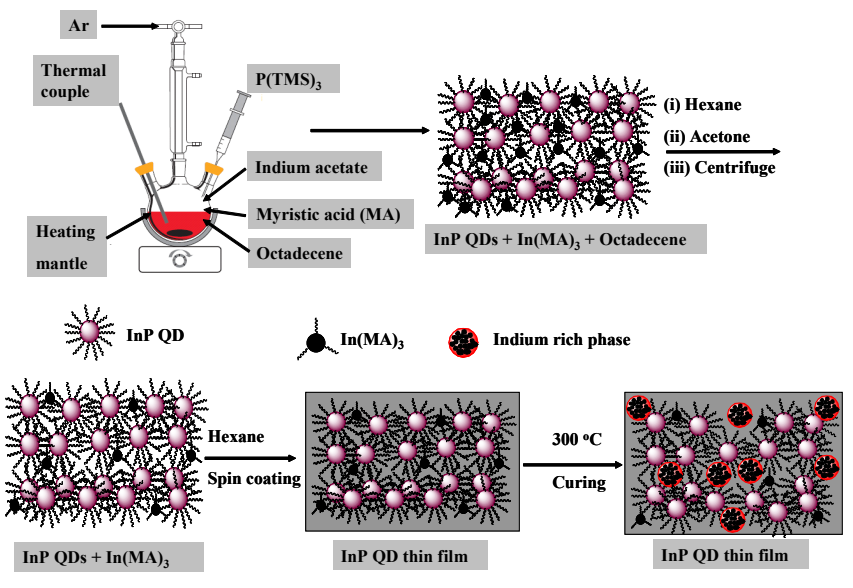

Figure 1. Illustration of synthesis of highly luminescent InP quantum dots (QDs) solution and quantum dot thin films. The InP QDs were stored in hexane prior to use for the spin coating process. The InP QDs prepared still contain some unreacted indium myristate.

It was earlier reported by Peng and coworkers that the concentration of ligand in a noncoordinating solvent can affect the reactivity of the metal precursor to balance between nucleation and growth during the formation of II-VI QDs. ${ }^{13}$ For InP QDs, the effects of concentration of ligand is more prominent than the cases of II-VI QDs. ${ }^{12}$

Figure 2 shows that when the molar ratio of indium to myristic acid is $1.0: 3.0$ or 1.0:3.5, distinct absorption peaks were observed, indicating that QDs are quite monodispersed. However, a more sharp and distinct absorption peak was obtained when the molar ratio of indium to myristic acid was 1.0:3.5, indicating that the QDs formed were more monodispersed than the QDs in which the molar ratio of indium to myristic acid was 1.0:3.0. So, for all further studies, quantum dots prepared from a molar concentration of indium to myristic acid 1.0:3.5 were employed. The average particle size of these quantum dots were estimated using the relationship between particle size and the first excitonic peak wavelength obtained from the absorption spectra. ${ }^{14}$ The estimated size was found to be approximately $4 \mathrm{~nm}$. When the molar ratio of indium to myristic acid changed to $1.0: 3.8$ or beyond, the absorption peak became a shoulder, indicating a broad size distribution. These results indicate that the concentration of ligand is also responsible for the formation of high quality InP QDs.

The photoluminescence (PL) spectra of the InP QD solution with different molar concentrations of indium to myristic acid are shown in the Fig. 3. The PL spectra of different samples match reasonably with the UV-visible spectra. When the molar ratio of indium to myristic acid was 1.0:3.8, multiple photoluminescence broad emission spectra were obtained, indicating that the QDs were no longer monodispersed, with different sizes formed in the solution.

An X-ray diffraction spectrum of InP QDs is shown in the Fig. 4, revealing the InP QDs formed to be polycrystalline and hexagonal. ${ }^{15}$ The diffraction peaks for the lattice planes $<111>$, $<220>$ were found at $2 \theta$ values of $26.8^{\circ}$ and $44.3^{\circ}$, respectively, which matches with that of the zinc blende structure of bulk InP crystal. There are additional peaks at the $2 \theta$ value of $19.5^{\circ}$, 
(a)

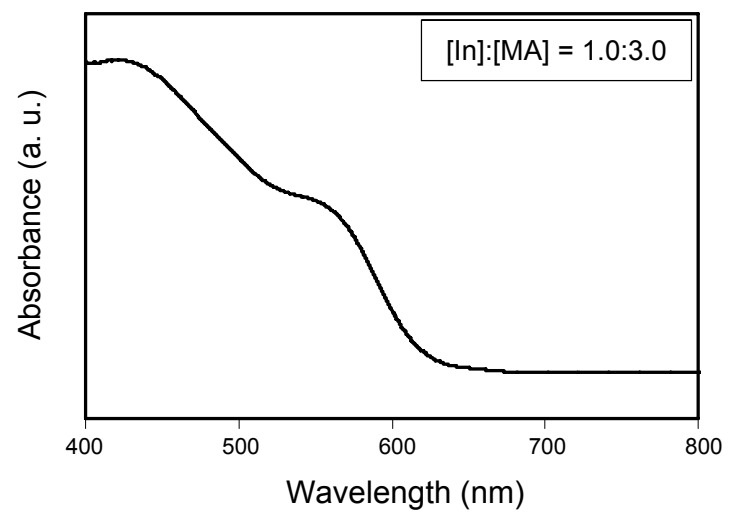

(b)

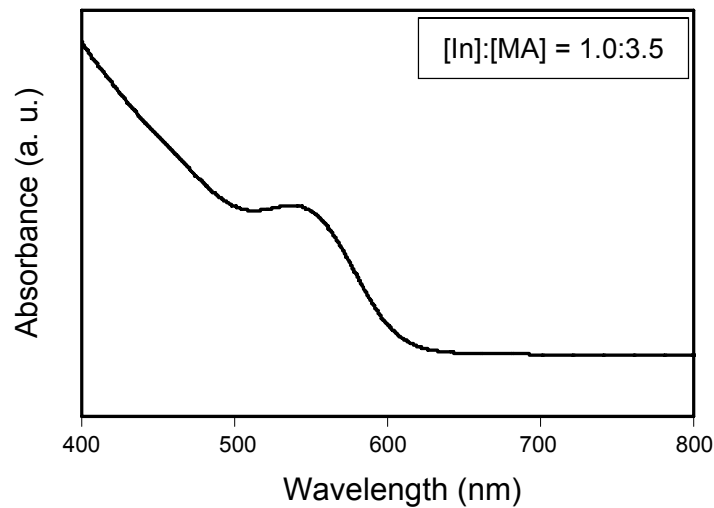

(c)

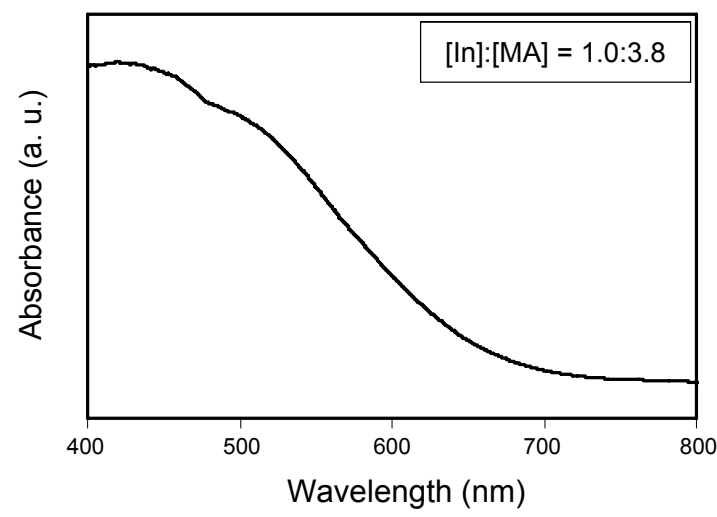

Figure 2. UV-visible spectra InP QD solutions in hexane prepared by varying the molar ratio of indium to myristic acid: (a) In:MA=1.0:3.0; (b) In:MA $=1.0: 3.5 ;$ (c) In:MA $=1.0: 3.8$.

which may be due to unreacted indium myristate present in the reaction mixture. ${ }^{16}$

A high-resolution transmission electron microscopy (HR TEM) image showing the formation of crystalline InP QDs is shown in Fig. 5. The QDs are generally spherically shaped, along with some elongated particles. The size of the QDs shown in the figure is approximately $4.2 \mathrm{~nm}$. For these QDs, the size determined by TEM was always slightly higher than that estimated from the absorption spectra. Most probably, this is due to the reason that oxide surface layers can be easily formed from small QDs, with a large surface area, which will be discussed in the following XPS and EDX results.

Synthesis and Characterization of InP QD Thin Films. By spin-coating the colloidal solution of InP QD onto a silicon wafer, InP QD thin films could be obtained (Fig. 1). To investi- (a)

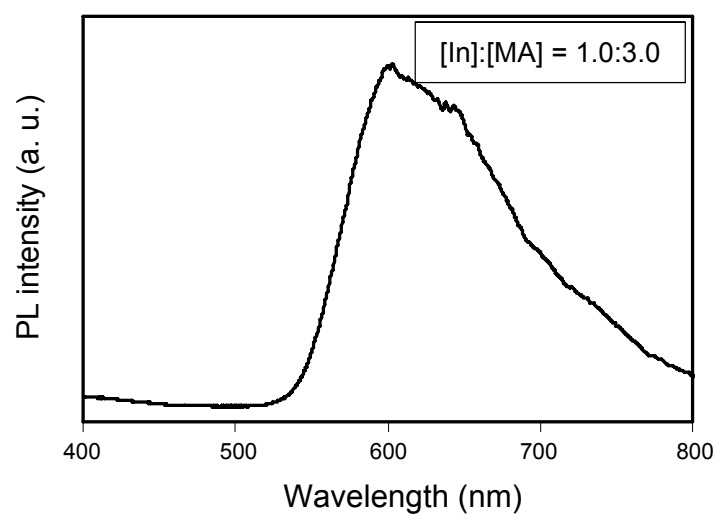

(b)

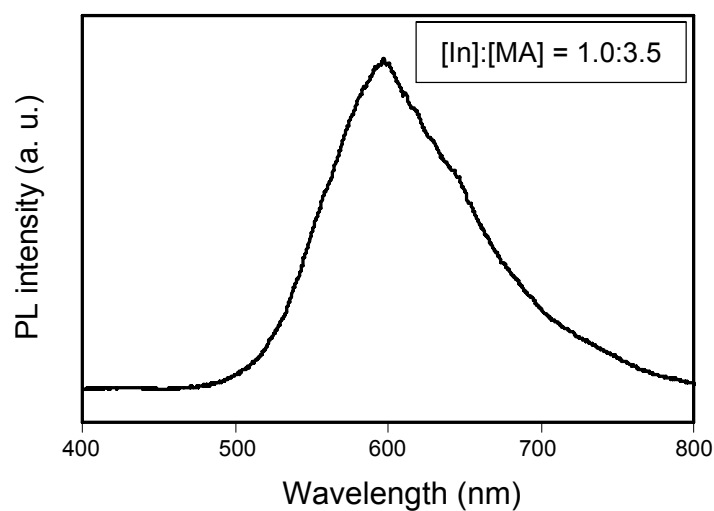

(c)

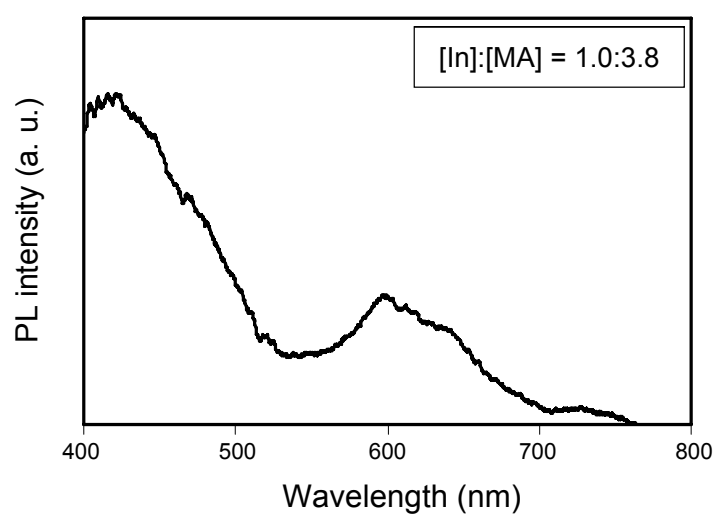

Figure 3. PL spectra InP quantum dot solutions in hexane prepared by varying the molar ratio of indium to myristic acid: (a) In:MA = 1.0:3.0; (b) In:MA = 1.0:3.5; (c) In:MA = 1.0:3.8.

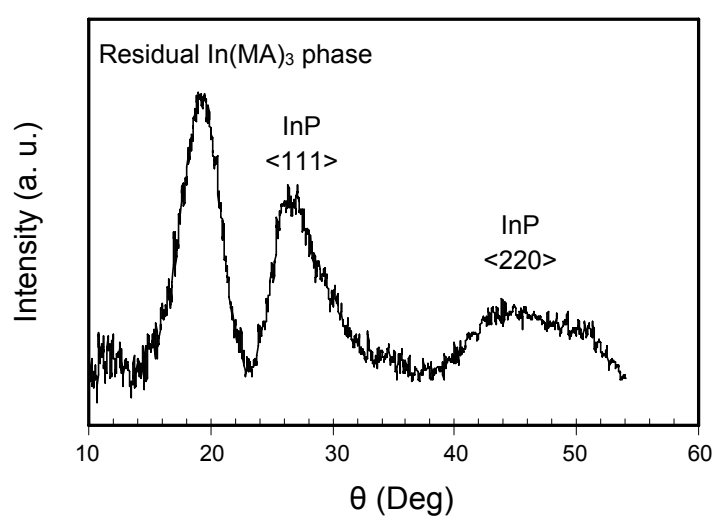

Figure 4. XRD pattern of InP QDs in hexane (molar ratio of indium to myristic acid of 1.0:3.5). 
gate the nanocrystaline phases in the InP QD thin films, TEM analysis was executed for samples cured at 60,200 , and $300{ }^{\circ} \mathrm{C}$, respectively, as shown in Fig. 6 . From the TEM images it was found that the thickness of the thin films cured at 60 and $200{ }^{\circ} \mathrm{C}$ were nearly identical (approximately $860 \mathrm{~nm}$ ), whereas at 300 ${ }^{\circ} \mathrm{C}$, the thickness of the thin film was found to be $760 \mathrm{~nm}$, indicating that the thickness of the thin films decreases with an increase in curing temperature, especially at $300{ }^{\circ} \mathrm{C}$. The decrease in thickness is mainly due to decomposition and elimination of the organic capping groups. An increase in curing

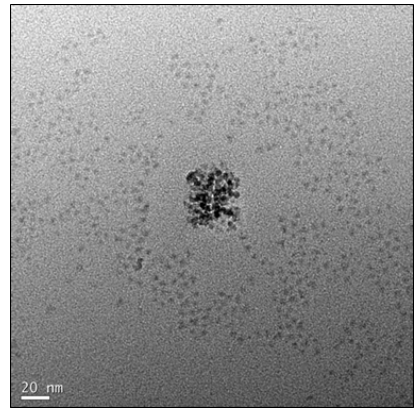

(a)

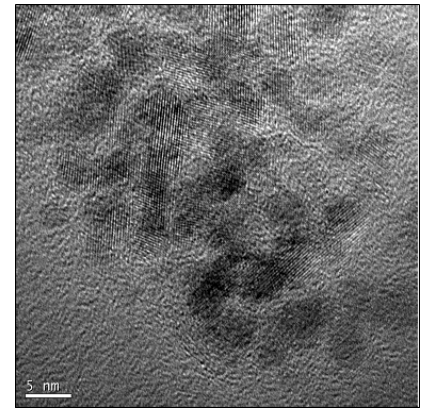

(b)

Figure 5. TEM and HRTEM images of InP QDs in hexane (molar ratio of indium to myristic acid of 1.0:3.5). The sample was prepared by placing a drop of colloidal quantum dot solution onto a carbon coated copper grid (300 mesh). The mean diameter of the particles in the TEM micrograph is $4.2 \mathrm{~nm}$.

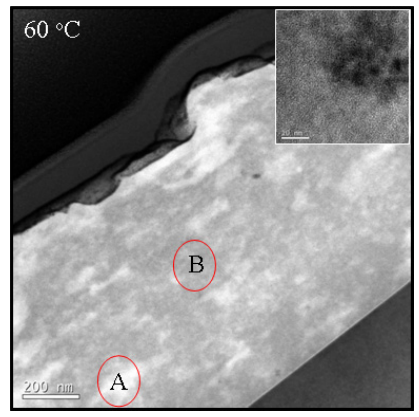

(a)

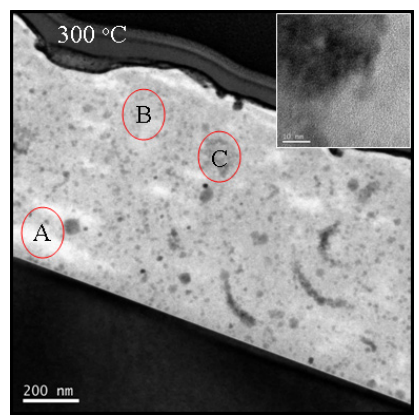

(c)

Figure 6. TEM images of InP QD thin films cured at: (a) $60{ }^{\circ} \mathrm{C}$; (b) $200{ }^{\circ} \mathrm{C}$; (c) $300{ }^{\circ} \mathrm{C}$. The films were prepared by spin-coating the colloidal QD solution onto a p-type silicon wafer. The molar ratio of indium to myristic acid in the QDs solution was 1.0:3.5. In the TEM images the circles "A", "B", and "C" represent the white, gray, and black regions. temperature is also expected to lead to interpenetration of the organic capping molecules, resulting in coupling between adjacent QDs in three-dimensional arrays. This lab observed different contrast regions in the TEM images of the QD thin films cured at different temperatures. To investigate the elemental composition at different contrast regions (A, B, C), EDX analysis was performed as shown in Fig. 7. In the TEM images, circles $\mathrm{A}, \mathrm{B}$, and $\mathrm{C}$ were assigned to white, gray, and black regions, respectively. From the EDX analysis, the white region (circle A) has much less concentration of indium and phosphorous relative to that of the gray region (circle B). However, in the white regions, the atomic concentrations of carbon and oxygen were much higher than the gray regions, indicating the presence of unreacted myristic acid or indium myristate. At $300{ }^{\circ} \mathrm{C}$ (Fig. 7(C)), some black regions (circle C) were observed, which contained higher concentrations of indium and phos-

(a)
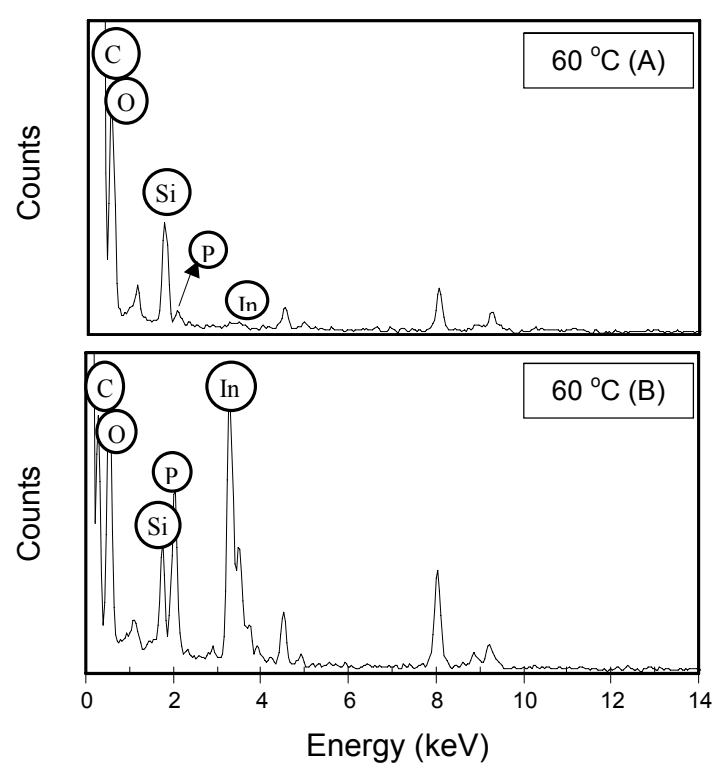

(b)
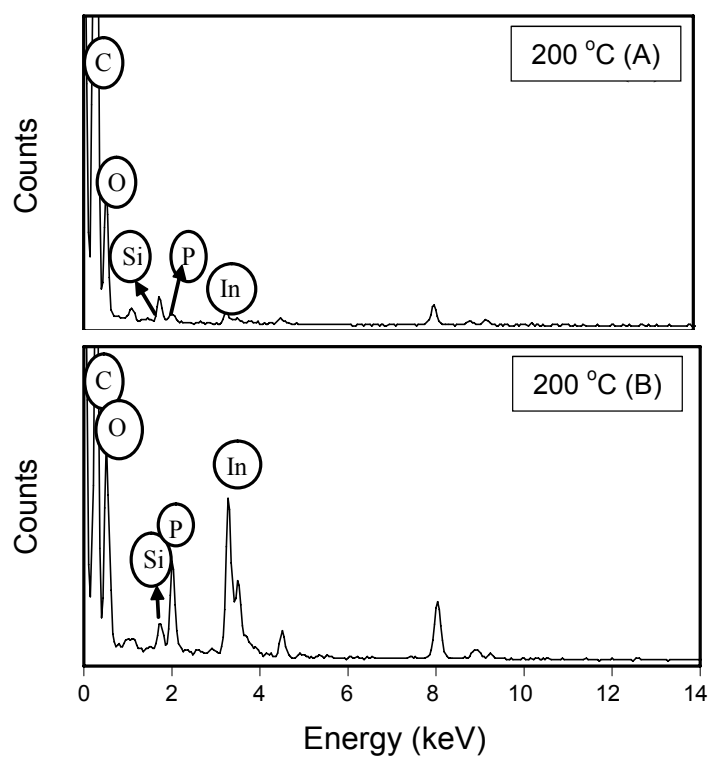

Figure 7. EDS spectrum measured at different regions ("A", "B", "C") of InP QD thin films cured at: (a) $60^{\circ} \mathrm{C}$; (b) $200{ }^{\circ} \mathrm{C}$; (c) $300^{\circ} \mathrm{C}$. 
(c)
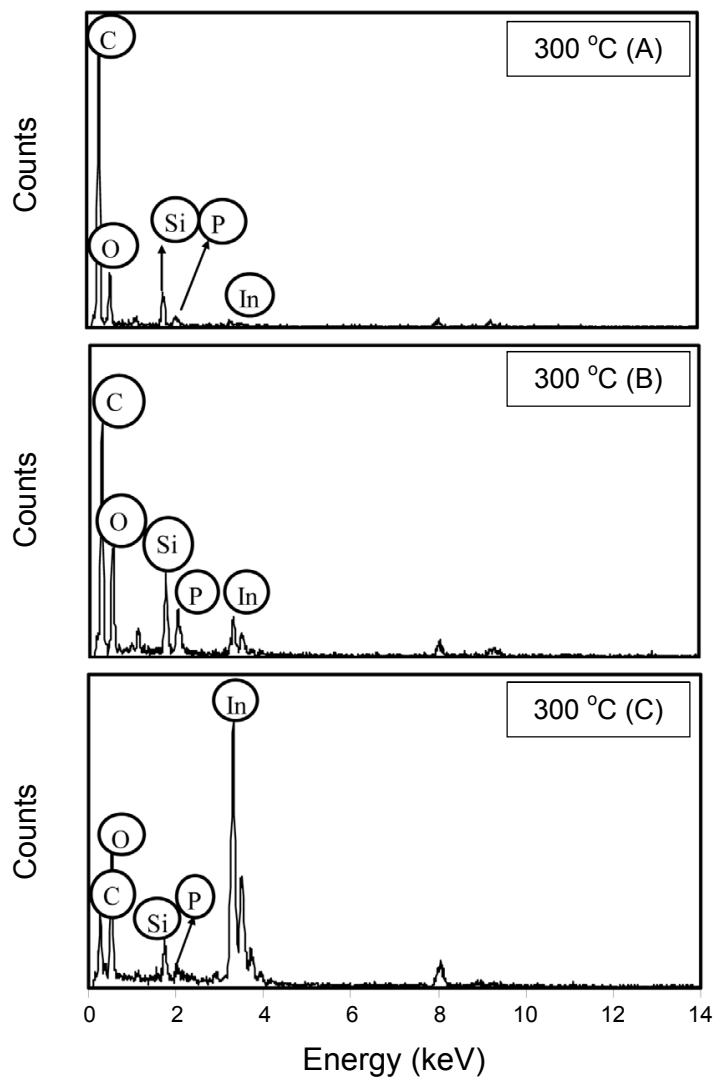

Figure 7. Continued.

phorous compared to the white and gray regions, formed as the result of unreacted indium myristate by decomposition of the organic ligand at $300{ }^{\circ} \mathrm{C}$. From these observations it was clear that the QDs still contained more unreacted indium myristate, as already seen in the XRD spectra. Close packing of QDs is necessary for studying the mechanism of quantum dot coupling and dielectric properties. Therefore, more size-selective precipitation is required to obtain QDs of high purity and uniform size, which is under study and will be reported later.

$\mathrm{X}$-Ray photoelectron spectroscopy was measured to determine the elemental composition of InP QD thin films cured at 60,200 , and $300{ }^{\circ} \mathrm{C}$. The high-resolution XPS (HRXPS) of each sample is shown in Fig. 8. From the figure are the photoelectron signals originating from the indium and phosphorous atoms. There are also peaks due to carbon and oxygen, arising from the organic ligands at the QD surface. Figure 8(d) shows the high-resolution spectra of the $\mathrm{P} 2 \mathrm{p}$ region of the InP QD thin film at three different temperatures. The presence of two peaks near 132.9 and $129.3 \mathrm{eV}$ were very much similar to previous results reported for InP QDs. ${ }^{16}$ It has been already reported by Guzelian that the peak near $129.3 \mathrm{eV}$ is due to phosphorous from InP QDs, and that the peak near $132.9 \mathrm{eV}$ is due mainly to oxidized phosphorous. ${ }^{5(\mathrm{~d})}$ Figure 8(c) shows the high-resolution spectrum of In $3 \mathrm{~d}$ region. The In $3 \mathrm{~d}$ core is spin-orbit split into $3 \mathrm{~d}_{5 / 2}$ and $3 \mathrm{~d}_{3 / 2}$ peaks. The $3 \mathrm{~d}_{5 / 2}$ peak position near $444.4 \mathrm{eV}$ and the $3 \mathrm{~d}_{3 / 2}$ peak position at approximately $452.1 \mathrm{eV}$ were well-matched with previously reported results for InP QDs. ${ }^{16}$

When the thin film was heated to $300{ }^{\circ} \mathrm{C}$, more phospho- (a)

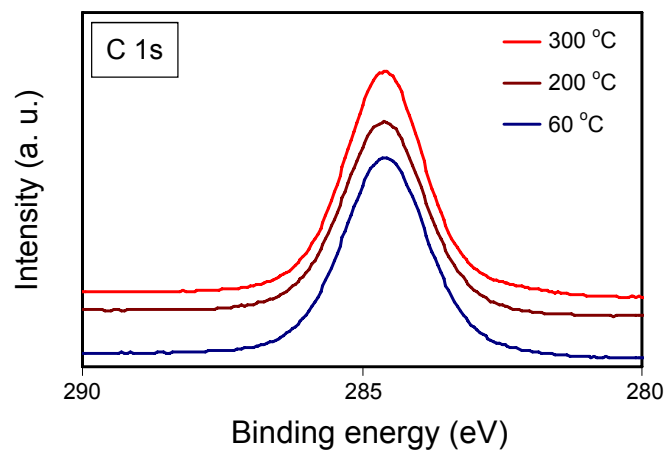

(b)

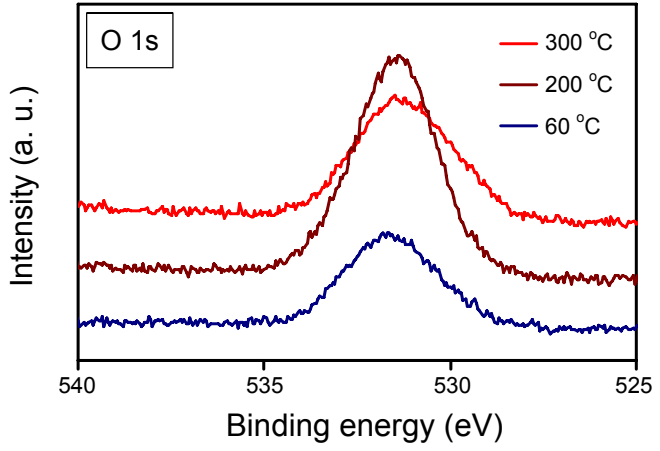

(c)

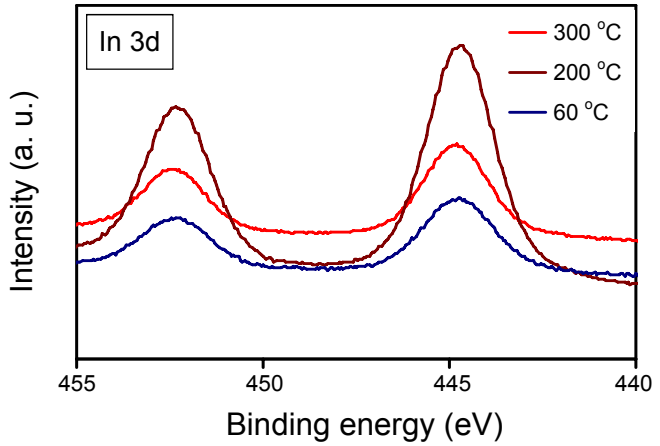

(d)

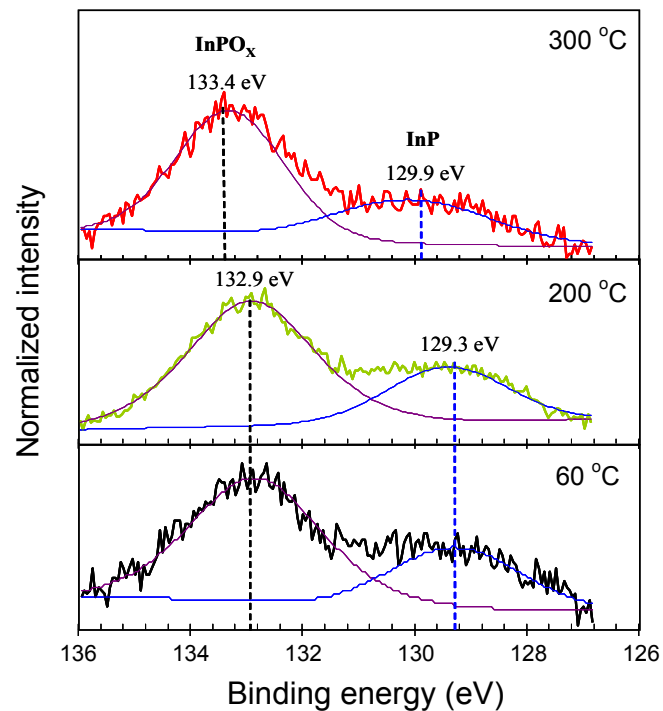

Figure 8. XPS quantification results of InP QD thin films cured at 60, 200 , and $300{ }^{\circ} \mathrm{C}$ : (a) HRXPS spectra of carbon 1s region; (b) HRXPS spectra of oxygen 1s region; (c) HRXPS spectra of indium 3d region; (d) normalized HRXPS spectra of phosphorous $2 p$ region. As curing temperature increases, the intensity of the peak at $129.2 \mathrm{eV}$ due to phosphorous atoms of the InP QDs decreases, indicating that more phosphorous from the InP QDs is oxidized. 
rous from the InP QD was oxidized, which can be seen from the Fig. 8 (d). Upon increasing the temperature from 60 to $300{ }^{\circ} \mathrm{C}$, the intensity of the peak at $129.3 \mathrm{eV}$, due to the phosphorous atoms of the InP QDs decreases, indicating that greater levels of phosphorous from InP QDs were oxidized. There is also considerable increase in chemical shift for both the peaks towards higher binding energy at $300{ }^{\circ} \mathrm{C}$. The higher shift in binding for the peak at $132.9 \mathrm{eV}$ may be attributed to stronger oxidation of the QD surface at higher temperature $\left(300^{\circ} \mathrm{C}\right)$. Due to the strong oxidation, new P-O bonds with higher binding energy are formed and numbers of $\mathrm{P}$ dangling bonds are decreased (passivated) causing also a shift of the peak at $129.3 \mathrm{eV}$ slightly towards higher binding energy. The increase in the rate of oxidation with the increase in curing temperature can also be verified from the EDS spectra of the QD thin films taken at different temperatures (Fig. 7). Due to higher oxidation, there was shrinkage of the inorganic core of the QD ${ }^{12}$, which resulted in a blue shift in the PL spectra as discussed below. Oxidation also passivates the QD surface by eliminating deep traps and by blocking nonradiative decays channels. This surface passivation greatly decreased the surface traps, resulting in increased luminescence.

The HRXPS spectra showed that irrespective of the curing temperature of the sample analyzed, the binding energies of indium, phosphorous, carbon, and oxygen were very much similar, which indicates the presence of InP QDs without giving a qualitative analysis of the purity of the sample.

Figure 9 shows the UV-visible spectra of the InP QD solutions and the thin films cured at 60,200 , and $300{ }^{\circ} \mathrm{C}$. For the thin films, the absorption peaks were slightly blue shifted with respect to the quantum dot solution spectra. Increase in the curing temperature also resulted in a marked blue shift and weakening of the first excitonic transition in the absorption spectra, indicating reduction in the inter QD coupling and a decrease in QD size due to oxidation. ${ }^{17}$

The photoluminescence (PL) studies performed on the QD solution and thin films cured at two different temperatures are shown in the Fig. 10. For the thin film cured at $300{ }^{\circ} \mathrm{C}$, a blue shift in the emission spectra was observed. This may be due to the decrease in core diameter of InP QDs by oxidation, as already explained by the XPS studies. However, at $300{ }^{\circ} \mathrm{C}$, the PL efficiency increased dramatically compared to the film cured at 60 and $200{ }^{\circ} \mathrm{C}$. This can be reasonably explained as follows. The low PL efficiency is usually due to insufficient passivation of the QD surface. A non-passivated InP QDs surface generally contains phosphorous $(\mathrm{P})$ and indium dangling bonds that result in the presence of surface traps for electrons or holes, providing nonradiative recombination pathways. ${ }^{18}$ However, it was reported earlier that presence of hole traps on the surface of the InP QDs are mainly due to P dangling bonds. ${ }^{19}$ This result indicates that $\mathrm{P}$ dangling bonds are mainly responsible for luminescence quenching for the samples at 60 and $200{ }^{\circ} \mathrm{C}$. At $300{ }^{\circ} \mathrm{C}$, the number of $\mathrm{P}$ dangling bonds decreases due to oxidation of the surface phosphorous atoms. Therefore, the drastic increase in photoluminescence efficiency is mainly due to better surface passivation of the InP QD surface by reduction of the number of $\mathrm{P}$ dangling bonds.

Electrical Properties of InP QD Thin Films. To investigate

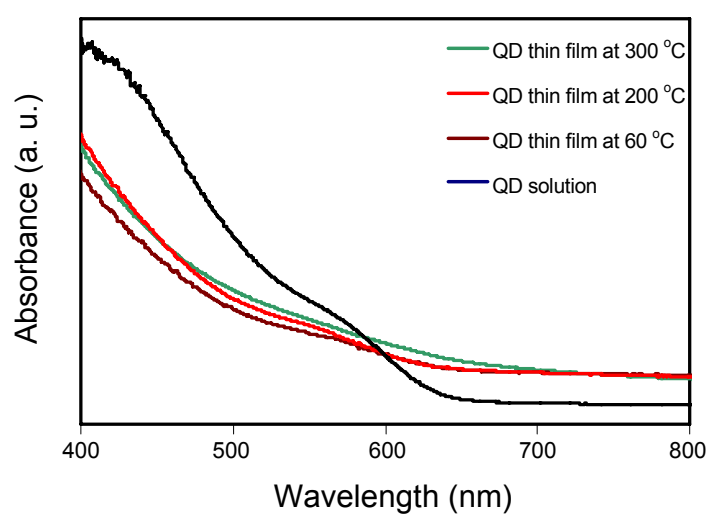

Figure 9. (a) UV-vis absorption spectra of the InP QD solution and InP QD thin films cured at 60,200 , and $300^{\circ} \mathrm{C}$. Indium to myristic acid molar ratio was 1.0:3.5 for all reactions.

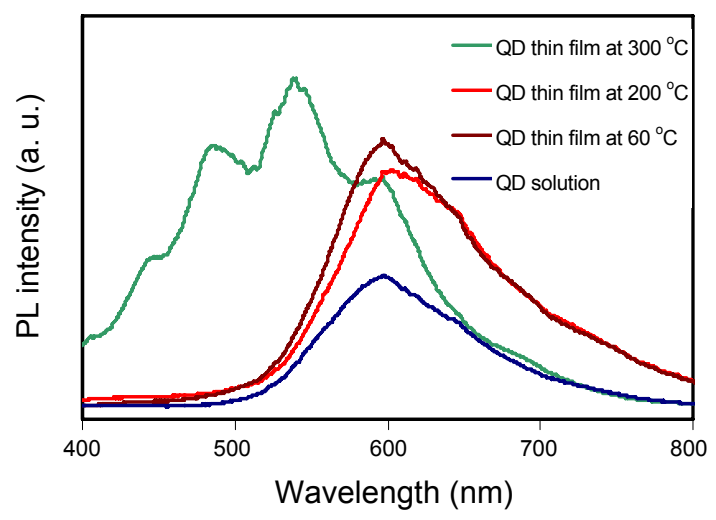

Figure 10. PL spectra of the InP QD solution and InP QD thin films cured at 60,200 , and $300^{\circ} \mathrm{C}$. Indium to myristic acid molar ratio was 1.0:3.5 for all reactions. As the curing temperature increased, the PL spectra showed a blue shift due to a decrease in the core diameter of the InP QD.

the charge trap properties of the InP QD thin films, capacitance-voltage $(\mathrm{C}-\mathrm{V})$ curves were measured in a typical metalinsulator-semiconductor (MIS) structure having thermallydeposited aluminum and low-doped p-type Si-wafer as a metal and insulator, respectively. The schematic representation of the above structure is shown in Fig. 11(a). The voltage of the top electrode was swept from -5 to $+5 \mathrm{~V}$ and back to $-5 \mathrm{~V}$.

Under the negative bias voltage at the top electrode, the holes in the Si substrate tend to move towards the interface between the insulator layer and the Si wafer. This condition is known as the "accumulation region" in typical C-V curves, as shown in Fig. 11(b). Similarly, when positive bias voltage is applied at the top electrode, the holes in the Si substrate tend to move down to the bulk of the silicon wafer. As a result, a depletion layer is formed at the interface between the insulator and silicon wafer. This condition is known as the "depletion region" in the $\mathrm{C}-\mathrm{V}$ curve, as shown in Fig. 11(b).

In the MIS structures, the band structure of the silicon substrate very close to the insulator is not flat, but rather bent down at the p-type semiconductor, as shown in the case of $\Phi_{\mathrm{M}}<\Phi_{\mathrm{SC}}$ (unbiased) in Fig. 11(b); this is known as band bending. When a negative bias voltage is applied at the top electrode, the band 
(a)

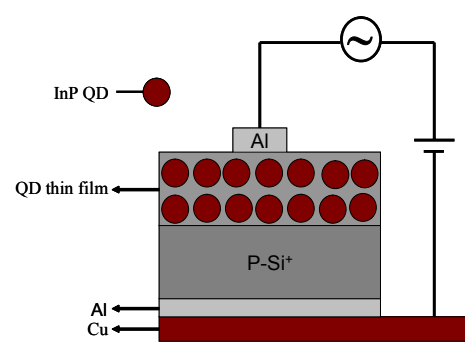

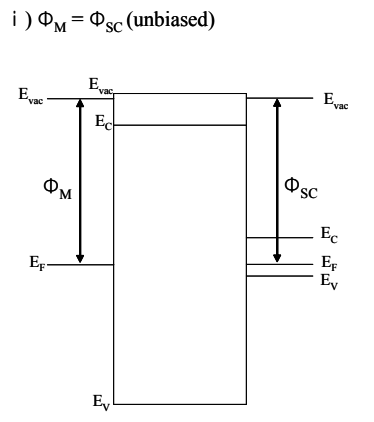

metal insulator semiconductor (b)

ii ) $\Phi_{\mathrm{M}}<\Phi_{\mathrm{SC}}($ unbiased $)$

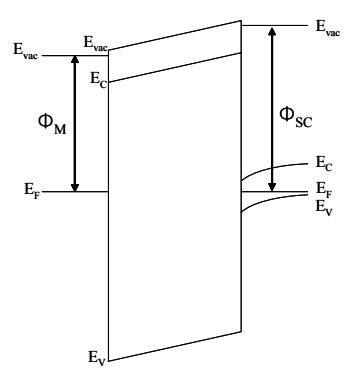

metal insulator semiconductor iii) $\Phi_{\mathrm{M}}<\Phi_{\mathrm{SC}}$ (flat band condition)

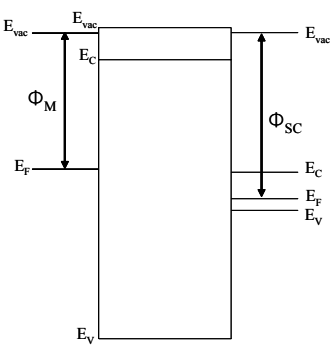

metal insulator semiconductor

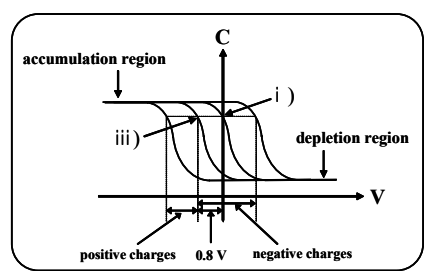

Figure 11. (a) Schematic representation of the MOS structure with the InP QD thin film; (b) Schematic representation of the C-V curve of an ideal p-type MOS structure.

(a)

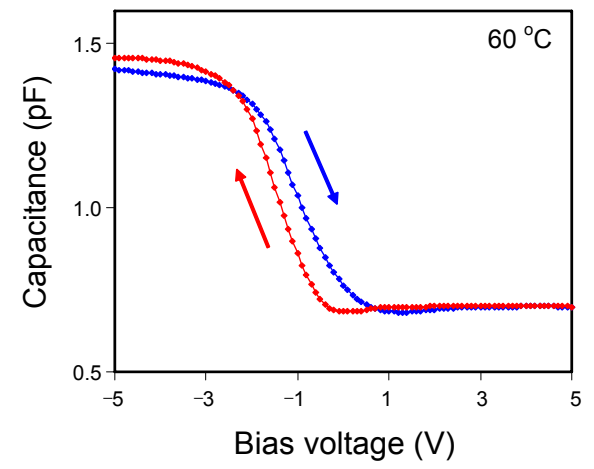

(b)

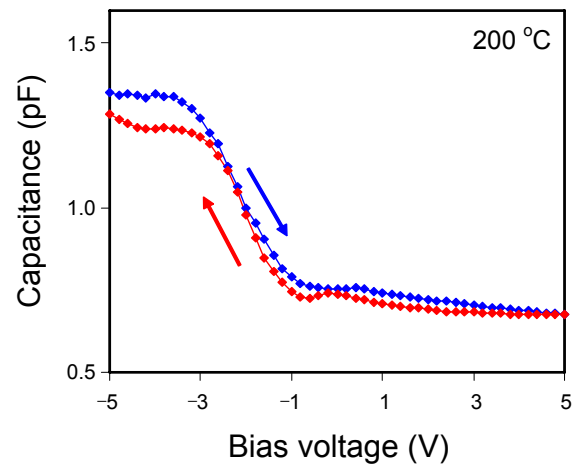

(c)

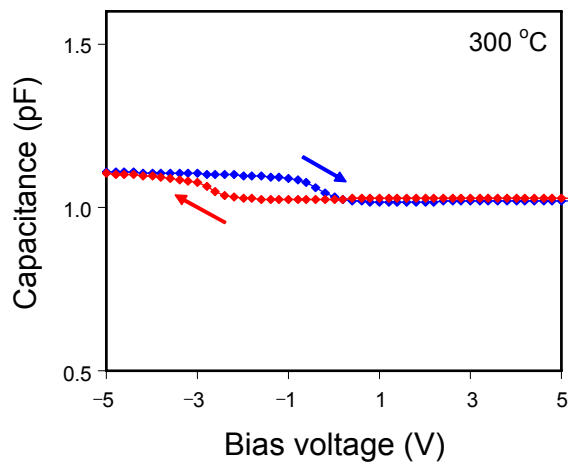

Figure 12. C-V characteristics of InP QD thin films cured at: (a) $60{ }^{\circ} \mathrm{C}$; (b) $200{ }^{\circ} \mathrm{C}$; (c) $300{ }^{\circ} \mathrm{C}$. Indium to myristic acid molar ratio was $1.0: 3.5$ for all reactions.

structures at the regions become flat, as shown in the case of $\Phi_{\mathrm{M}}<\Phi_{\mathrm{SC}}$ (flat band condition) of the Fig. 11(b). In the MIS structure containing an aluminum electrode and p-type semiconductor, $\Delta \mathrm{V}_{\mathrm{fb}}$ is estimated to be $0.8 \mathrm{eV}$, as the Fermi energy of $4.1 \mathrm{eV}$ of aluminum $\left(\Phi_{\mathrm{M}}\right)$ is lower than $4.9 \mathrm{eV}$, that of the semiconductor $\left(\Phi_{\mathrm{SC}}\right)$.

However, the presence of some charges, positive or negative, in the insulator can also change the extent of band bending. As a result, in order to make the band flat, the voltage applied at the aluminum electrode has to be different, which means that the $\Delta \mathrm{V}_{\mathrm{fb}}$ value is changed. Generally, the presence of positive charges shifts the $\Delta \mathrm{V}_{\mathrm{fb}}$ in the negative direction while the presence of negative charges shifts the $\Delta \mathrm{V}_{\mathrm{fb}}$ in a positive direction.

Figure 12 shows the $\mathrm{C}-\mathrm{V}$ curves of the InP QD thin films at three different curing temperatures. At 60 and $200{ }^{\circ} \mathrm{C}$, there is only a negative $\Delta \mathrm{V}_{\mathrm{fb}}$, indicating the presence of positive charges in the QD thin films. In addition, for the two curing temperatures, the $\Delta \mathrm{V}_{\mathrm{fb}}$ values for the reverse sweep direction are not very different from those for the forward sweep direction, as summarized in Table 1. This weak hysteresis behavior of the charge trapping indicates that the positive charges exist mainly as intrinsic ones in the InP QD thin films, which are neither injected nor detrapped from/to the aluminium gate electrode or

Table 1. The values of flat band $\operatorname{shift}\left(\Delta \mathrm{V}_{\mathrm{fb}}\right)$ under forward and reversed biased conditions estimated from the $\mathrm{C}-\mathrm{V}$ curves of InP quantum dot thin films cured at 60,200 , and $300{ }^{\circ} \mathrm{C}$.

\begin{tabular}{ccc}
\hline Curing temp. & $\Delta \mathrm{V}_{\mathrm{fb}}$ (reverse) & $\Delta \mathrm{V}_{\mathrm{fb}}$ (forward) \\
\hline $60{ }^{\circ} \mathrm{C}$ & $-1.92 \mathrm{~V}$ & $-1.56 \mathrm{~V}$ \\
$200{ }^{\circ} \mathrm{C}$ & $-2.43 \mathrm{~V}$ & $-2.71 \mathrm{~V}$ \\
$300^{\circ} \mathrm{C}$ & $-2.86 \mathrm{~V}$ & $-0.71 \mathrm{~V}$ \\
\hline
\end{tabular}


(1)
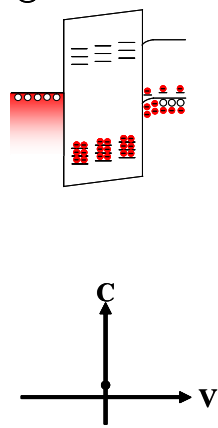

(5)
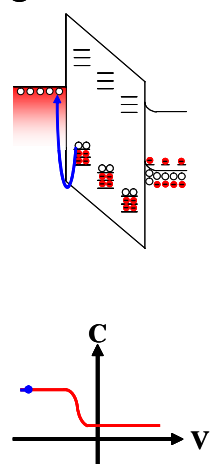
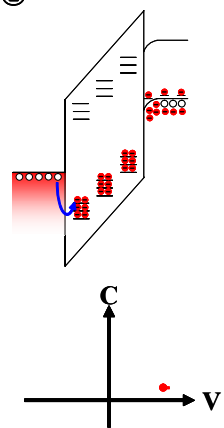

(6)

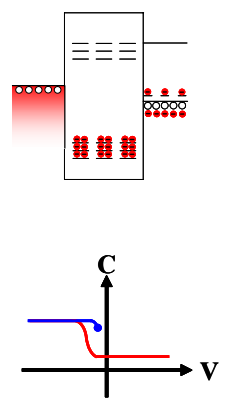

(3)
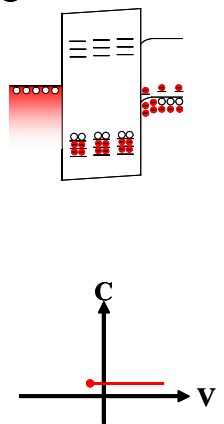

(7)

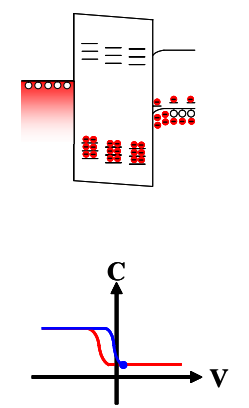

(4)

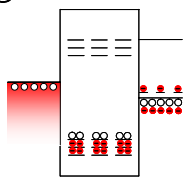

(8)

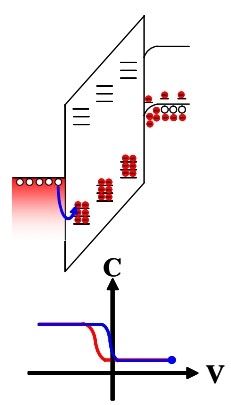

Figure 13. The tentative model of trapping and detrapping of holes explaining the distinct clockwise hysteresis observed for the film cured at $300{ }^{\circ} \mathrm{C}$. The energy levels of the low HOMO-LUMO gap appearing in the insulator layer is thought to originate from the indium-rich phases, the presences of which was proposed as the black regions (circle $\mathrm{C}$ ) in the EDX results.

the silicon substrate as the voltages are applied. What originates these intrinsic positive charges in the InP QD thin films? The authors speculate that $\mathrm{P}$ dangling bonds on the InP QD surface generate the intrinsic positive charges. This is due in part to: (1) Nozik and Thurnauer et al. concluded ${ }^{19}$ that a deep hole trap center ( $g=0.58$ in electron paramagnetic resonance) at the interface of the InP QDs was attributed to P dangling bonds; (2) the authors' assertion above that the relatively low PL efficiency for the 60 and $200{ }^{\circ} \mathrm{C}$ was attributed to the $\mathrm{P}$ dangling bonds of the InP QDs surfaces.

In order to maintain logical consistency of above reasoning, the possibility of existence of those intrinsic positive charges has to be abandoned for the case of the $300{ }^{\circ} \mathrm{C}$ curing temperature. This is because the number of $\mathrm{P}$ dangling bonds decreases due to oxidation of surface phosphorous atoms at $300{ }^{\circ} \mathrm{C}$, resulting in a drastic increase in PL efficiency, as discussed in the above XPS and PL results. This conjecture is confirmed in the $\mathrm{C}-\mathrm{V}$ curves of large clockwise hysteresis for the $300{ }^{\circ} \mathrm{C}$ thin film, as shown in Fig. 11(c) and Table 1, whereby the $\Delta V_{\mathrm{fb}}$ value $(-2.86 \mathrm{~V})$ for the reverse sweep direction is distinctively different from that $(-0.71 \mathrm{~V})$ of the forward sweep direction. The observed clockwise hysteresis can be explained by the trapping and detrapping of positive charges. Under positive bias conditions, holes are injected into the QD thin film from the top aluminum electrode, generating the negative $\Delta \mathrm{V}_{\mathrm{fb}}$ in reverse sweep direction, as described schematically in Fig. 13. As a negative voltage is applied at the top aluminum electrode, the holes in the InP QD thin films are detrapped into the top alumi- num electrode, providing a quite smaller negative $\Delta \mathrm{V}_{\mathrm{fb}}$ in the forward sweep direction, also shown in Fig. 13.

What is responsible for the trapping and detrapping of the holes in the InP QD thin films cured at $300{ }^{\circ} \mathrm{C}$ ? The authors already asserted that the number the $\mathrm{P}$ dangling bonds on the InP QD surface decreases for the case of $300^{\circ} \mathrm{C}$. Thus, another physic-chemical origin is required to explain the positive charge trapping phenomena. Existence of the indium-rich phases for the $300{ }^{\circ} \mathrm{C}$ thin films that were observed in the above EDX results can be a clue for that question. It is plausible that the HOMO-LUMO gap of the indium-rich phases is lower than the band gap of the surrounding dielectric medium that consists of InP QDs and unreacted myristic acids or indium myristate. Accordingly, the indium-rich phases are thought to act as deep centers for hole trapping between the conduction and valence bands of the InP QD thin films. The energy levels of the deep centers are schematically drawn in Fig. 13.

\section{Conclusions}

Highly luminescent InP QDs with controlled size and size distribution were synthesized in a non-coordinating solvent. All InP QD solids in the form thin film were fabricated from colloidal solutions by a spin-coating method. The optical properties of the QD thin films were studied for the film curing temperatures of 60,200 , and $300{ }^{\circ} \mathrm{C}$. An enhancement in the PL intensity was achieved at $300{ }^{\circ} \mathrm{C}$ by minimizing defects due to phosphorous dangling bonds. However, a blue shift was 
observed in the PL spectra at $300{ }^{\circ} \mathrm{C}$ due to greater oxidation of InP as confirmed by XPS. This is thought to be due to the formation of a core shell structure. The electrical properties of the thin films were also studied by $\mathrm{C}-\mathrm{V}$ measurements on a MIS device. At 60 and $300{ }^{\circ} \mathrm{C}$, negative flat band shifts $\left(\Delta \mathrm{V}_{\mathrm{fb}}\right)$ were observed, which were explained by the presence of $\mathrm{P}$ dangling bonds on the InP QD surface. At $300{ }^{\circ} \mathrm{C}$, clockwise hysteresis was observed due to trapping and detrapping of positive charges on the thin film, which can be explained by proposing deep energy levels due to indium-rich phases.

Acknowledgments. This research was supported by Basic Science Research Program through the National Research Foundation of Korea (NRF) funded by the Ministry of Education, Science and Technology (No. 2010-0008824).

\section{References}

1. (a) Klein, D. L.; Roth, R.; Lim, A. K. L.; Alivisatos, A. P.; McEuen, P. L. Nature 1997, 389, 699. (b) Klimov, V. I.; Mikhailovsky, A. A.; Xu, S.; Malko, A.; Hollingsworth, J. A.; Leatherdale, C. A.; Eisler, H. J.; Bawendi, M. G. Science 2000, 290, 314. (c) Zhao, J.; Zhang, J.; Jiang, C.; Bohnenberger, J.; Basche, T.; Mews, A. J. Appl. Phys. 2004, 96, 3206.

2. (a) Michalet, X.; Pinaud, F. F.; Bentolila, L. A.; Tsay, J. M.; Doose, S.; Li, J. J.; Sundaresan, G.; Wu, A. M.; Gambhir, S. S.; Weiss, S. Science 2005, 307, 538. (b) Medinitz, I. L.; Uyeda, H. T.; Goldman, E. R.; Mattoussi, H. Nat. Mater. 2005, 4, 435. (c) Alivisatos, A. P.; Gu, W.; Larabell, C. Annu. Rev. Biomed. Eng. 2005, 7, 55. (d) Dahan, M.; Levi, S.; Luccardini, C.; Rostaing, P.; Riveau, B.; Triller, A. Science 2003, 302, 442. (e) Jaiswal, J. K.; Mattoussi, H.; Mauro, J. M.; Simon, S. M. Nat. Biotechnol. 2003, 21, 47. (f) Kim, S.; Lim, Y. T.; Soltesz, E. G.; De Grand, A. M.; Lee, J.; Nakayama, A.; Parker, J. A.; Mihaljevic, T.; Laurence, R. G.; Dor, D. M.; Cohn, L. H.; Bawendi, M. G.; Frangioni, J. V. Nat. Biotechnol. 2004, 22, 93 .

3. (a) Huynh, W. U.; Dittmer, J. J.; Alivisatos, A. P. Science 2002, 295, 2425. (b) McDonald, S. A.; Konstantatos, G.; Zhang, S.; Cyr, P. W.; Klem, E. J. D.; Levina, L.; Sargent, E. H. Nat. Mater. 2005, 4,138 .

4. (a) Hines, M. A.; Guyot-Sionnest, P. J. Phys. Chem. 1996, 100, 468. (b) Peng, X.; Schlamp, M. C.; Kadavanich, A. V.; Alivisatos, A. P. J. Am. Chem. Soc. 1997, 119, 7019. (c) Talapin, D. V.; Rogach, A. L.; Kornowski, A.; Haase, M.; Weller, H. Nano Lett. 2001, 1, 207. (d) Lim, J.; Jun, S.; Jang, E.; Baik, H.; Kim, H.; Cho, J. Adv. Mater. 2007, 19, 1927.

5. (a) Talapin, D. V.; Rogach, A. L.; Mekis, I.; Haubold, S.; Kornowski, A.; Haase, M.; Weller, H. Colloids Surf., A. 2002, 202, 145. (b) Micic, O. I.; Ahrenkiel, S. P.; Nozik, A. J. Appl. Phys. Lett. 2001, 78, 4022. (c) Li, Y. D.; Duan, X. F.; Qian, Y. T.; Yang, L.; Ji, M. R.; Li, C. W. J. Am. Chem. Soc. 1997, 117, 7869. (d) Guzelian, A. A.; Katari, J. E. B.; Kadavanich, A. V.; Banin, U.; Hamad, K.; Juban, E.; Alivisatos, A. P.; Wolters, R. H.; Arnold, C. C.; Heath, J. R. J. Phys. Chem.1996, 100, 7212. (e) Micic, O. I.; Sprague, J. R.; Curtis, C. J.; Jones, K. M.; Machol, J. L.; Nozik, A. J.; Giessen, H.; Fluegel, B.; Mohs, G.; Peyghambarian, N. J. Phys. Chem. 1995, 99, 7754. (f) Micic, O. I.; Curtis, C. J.; Jones, K. M.; Sprague, J. R.; Nozik, A. J. J. Phys. Chem. 1994, 98, 4966.

6. (a) Battagila, D.; Peng, X. Nano Lett. 2002, 2, 1027. (b) Xie, R.; Battagila, D.; Peng, X. J. Am. Chem. Soc. 2007, 129, 15432.

7. Shevchenko, E. V.; Talapin, D. V.; Kotov, N. A.; O'Brien, S.; Murray, C. B. Nature 2006, 439, 55.

8. Liu, G. F.; Sroubek, Z.; Yarmoff, J. A. Phys. Rev. Lett. 2004, 92, 216801.

9. (a) Seon, J.; Lee. S. Kim J. M.; Jeong, H. D. Chem. Mater. 2009, 21, 604. (b) Choi, J. K.; Jang, S.; Sohn, H.; Jeong, H. D. J. Am. Chem. Soc. 2009, 131, 17894.

10. (a) Woodall, J. M. Science 1980, 208, 908. (b) Kogelnik, H. Science 1985, 228, 1043.

11. Micic, O. I.; Jones, K. M.; Cahil, A.; Nojik, A. J. J. Phys. Chem. 1998, 102, 9791

12. Xie, R.; Battaglia, D.; Peng, X. G. J. Am. Chem. Soc. 2007, 129, 15432.

13. Yu, W. W; Peng, X. Angew. Chem. Int. Ed. Engl. 2002, 41, 2368.

14. Li, C.; Ando, M.; Enomoto, H.; Murase, N. J. Phys. Chem. C 2008, 112, 20190.

15. Aksoy, F.; Kayali, R.; Oztas, M.; Bedir, M. J. Phys. and Chem. Solids 2008, 69, 835.

16. Lucey, D. W.; Macrae, D. J.; Furis, M.; Sahoo, Y.; Cartwright, A. N.; Prasad, P. N. Chem. Mater. 2005, 17, 3754.

17. Luther, M. J.; Law, M.; Song, Q.; Perkins, C. L.; Beard, M. C.; Nozik, A. J. Acs Nano. 2008, 2, 271.

18. Qu, L.; Peng, X. J. Am. Chem. Soc. 2002, 124, 2049.

19. Micic, O. I.; Nozik, A. J.; Lifshitz, E.; Rajs, T.; Poluektov, O. G.; Thurnauer, M. C. J. Phys. Chem. B 2002, 106, 4390. 